\title{
Modelling of load transfer between porous matrix and short fibres in ceramic matrix composites
}

\author{
J. G. P. Silva ${ }^{1,2}$, D. Hotza ${ }^{1}$, R. Janssen ${ }^{2}$ \& H. A. Al-Qureshi ${ }^{1}$ \\ ${ }^{1}$ Federal University of Santa Catarina (UFSC), Florianópolis, SC, Brazil \\ ${ }^{2}$ Technical University of Hamburg-Harburg(TUHH), Germany
}

\begin{abstract}
The aim of this work is to present a model of load transfer between porous matrix and short fibres in ceramic matrix composites. This analysis is based on the earlier shear-lag models used for polymeric composites. However, geometry and strength of fibres in addition to the matrix porosity are included in the present analysis. The theoretical curves for the longitudinal and shear stresses distributions along the fibre-porous matrix interface are presented. They exhibited a maximum strength point at the middle of the short fibres. It became evident that the critical length is governed by the relative properties of the fibres, matrix and porosity, which greatly influenced the load carrying capacity of the fibres in the composites. In addition, the present simplified solution facilitates the understanding of the interface mechanism using porous matrix.

Keywords: modelling, ceramic matrix composites, shear-lag models, porous ceramics.
\end{abstract}

\section{Introduction}

Modern structural ceramic composites possess a number of unique properties that cannot be achieved by other materials. Therefore, they have a potential for saving energy, reducing wear, and increasing the lifetime of components [1]. However, regardless of their remarkable properties, structural ceramics are not as widely used in industry as they should and could be. Among the reasons for the reluctance of industry to introduce structural ceramic as components are [1]:

- high price of ceramic parts,

- insufficient knowledge in "traditional" metal-oriented engineering, 
- low toughness,

- need of redesigning components to meet specific requirements.

In the case of ceramic composites, the price factor is still the major limitation in terms of commercial applications. This is especially true for components made of composites with complex structures and requiring mechanical performance and reliability. To this aim, fibre-reinforced ceramic composites have been developed to overcome the fragility and unreliability of monolithic ceramics. Their main advantages are high-temperature resistance, low density, better corrosion resistance and adequate damage tolerance [2].

There is a wide range of fibre-reinforced ceramic composites depending on the chemical composition of the matrix and reinforcement, although currently only $\mathrm{C}_{\mathrm{f}}-\mathrm{C} / \mathrm{SiC}$ composites produced by silicon infiltration have reached commercial production. However, fibre-reinforced ceramic composites based on oxides (alumina, mullite) can provide key benefits about long-term stability under oxidizing atmospheres [2].

Despite considerable interest in oxide ceramic composites in the past decade [3-5], there is still no production concept that meets the requirements in view of cost and performance.

This study aims to investigate the load transfer phenomenon in ceramic-ceramic short fibre composites, using a simplified mathematical model that aims to predict the actual effectiveness of reinforcement depending on the material properties.

\section{Modelling}

\subsection{Previous considerations and analysis}

Considering a loaded composite made of a dense fibre with length $2 L$ embedded in a porous matrix made of the same material of the fibre, hereby is assumed that no slippage occurs between fibre and matrix. It should be also considered that the Poisson's ratio of fibre and matrix is the same, which implies on the inexistence of transversal stress when the loading is applied along the fibre. Considering the displacements in the fibre $u$ and far away from the fibre $v$ : (Fig. 1):

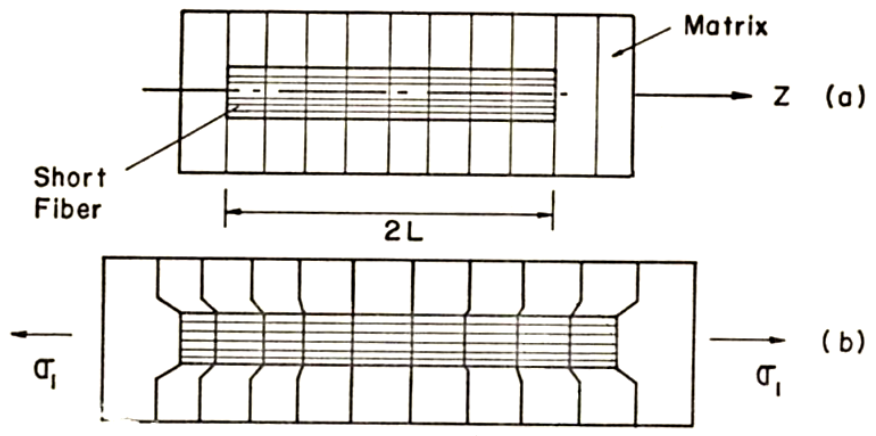

Figure 1: Simplified scheme of the stress field around the fibre. (a) without loading; (b) loaded [6]. 
From Hooke's law:

$$
\sigma=E \varepsilon=\frac{P}{A}=E \frac{\delta}{L}
$$

Taking the differential:

$$
\frac{d P}{d x}=\frac{E A}{L} \cdot \frac{d \delta}{d x}
$$

Cox proposes a similar behaviour [7]:

$$
\frac{d P_{f}}{d x}=B(u-v)
$$

where $P_{\mathrm{f}}$ is the load acting on the fibre and $B$ is a constant that depends on the fibre distribution and the Young's Modulus of fibre and matrix.

Differentiation of Eq. (3) leads to:

$$
\frac{d^{2} P_{f}}{d x^{2}}=B\left(\frac{d u}{d x}-\frac{d v}{d x}\right)
$$

The derivatives of $u$ and $v$ can be taken as the deformations in the fibre and matrix, respectively:

$$
\begin{gathered}
\frac{d u}{d x}=\frac{P_{f}}{A_{f} E_{f}} \\
\frac{d v}{d x}=\varepsilon
\end{gathered}
$$

Substitution of (5) and (6) in (4), gives:

$$
\frac{d^{2} P_{f}}{d x^{2}}=B\left(\frac{P_{f}}{A_{f} E_{f}}-\varepsilon\right)
$$

A solution to this differential equation leads to:

$$
P_{f}=E_{f} A_{f} \varepsilon+S \sinh (\beta x)+T \cosh (\beta x)
$$

where: 


$$
\beta=\sqrt{\frac{B}{E_{f} A_{f}}}
$$

and $\mathrm{S}$ and $\mathrm{T}$ are constants defined by the boundary conditions.

To simplify the solution, instead of the stress distribution proposed by Cox, a model proposing that the stress drop in the extremity of the fibre follows a quadratic behaviour.

To evaluate the model herein described, it is possible to apply the equations to a model composite, made of a porous alumina matrix and alumina fibres. Table 1 summarizes the properties that are relevant for the calculation.

Table 1: $\quad$ Properties of the model composite.

\begin{tabular}{|c|c|}
\hline Property & Value \\
\hline Fibre volume fraction & 0.45 \\
\hline Matrix porosity fraction & 0.24 \\
\hline Fibre length $-21(\mathrm{~mm})$ & 50.8 \\
\hline Fibre diameter $(\mu \mathrm{m})$ & 10 \\
\hline Ratio Critical length/Length or $\alpha$ & 0.25 \\
\hline
\end{tabular}

\subsection{Proposed model}

According to Fig.2, let us consider a composite with fibres whose length is $2 L$, diameter $2 r$ and Young's modulus $E_{\mathrm{f}}$, embedded in a matrix with porosity $\rho$, made of the same material of the fibre. Hereby we define the critical length $L_{\mathrm{c}}$, in which from the tip of the fibre the stress distribution isn't constant by the shearlag between matrix and fibre. It is more feasible to work with $\alpha$, the ratio between the critical length and fibre length, being $L_{c}=\alpha \cdot L$.

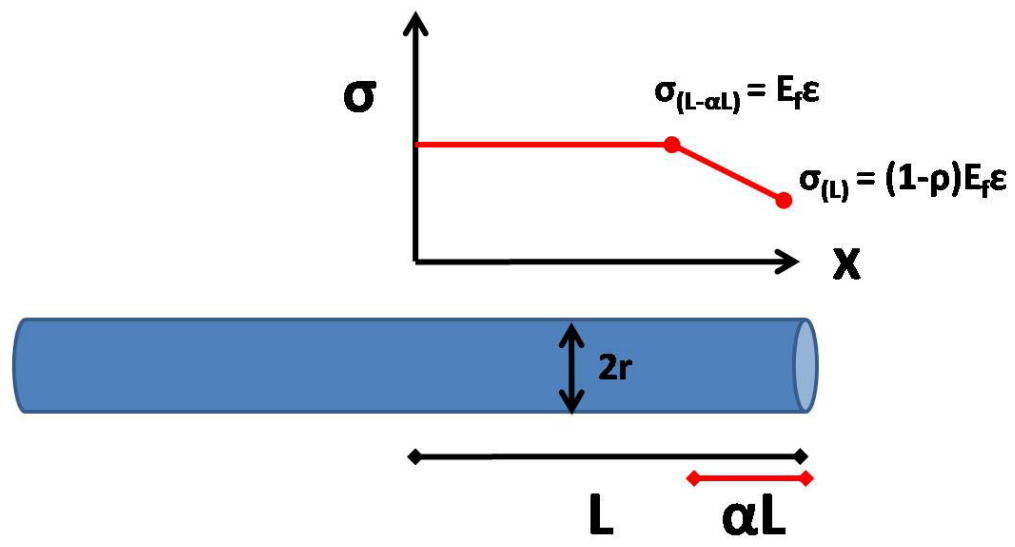

Figure 2: $\quad$ Proposed stress distribution and boundary conditions. 
It can be proposed that the stress distribution between the points $L-\alpha L$ and $L$ follows a behaviour such as:

$$
\sigma_{f}(x)=A x+B
$$

By using the boundary conditions given in Fig. 2, and substituting them in (10):

$$
\begin{aligned}
& E_{f} \varepsilon=A(L-\alpha L)+B \\
& (1-\rho) E_{f} \varepsilon=A L+B
\end{aligned}
$$

Isolating $B$ in (12) and replacing in (11):

$$
\begin{gathered}
(1-\rho) E_{f} \varepsilon-A L=E_{f} \varepsilon-A(L-\alpha L) \\
-\rho E_{f} \varepsilon=A \alpha L
\end{gathered}
$$

And then:

$$
A=-\frac{\rho E_{f} \varepsilon}{\alpha L}
$$

By replacing $A$ from (12):

$$
(1-\rho) E_{f} \varepsilon=-\frac{\rho E_{f} \varepsilon}{\alpha}+B
$$

Therefore, $B$ is given by:

$$
B=\left[1-\rho\left(1-\frac{1}{\alpha}\right)\right] E_{f} \varepsilon
$$

By replacing the constants in (10), we have the stress distribution behaviour:

$$
\sigma_{f}(x)=-\frac{\rho E_{f} \varepsilon}{\alpha L} x+\left[1-\rho\left(1-\frac{1}{\alpha}\right)\right] E_{f} \varepsilon
$$

To determine the shear stresses along the fibre, the force equilibrium in a fibre element with diameter $2 r$ and length $d x$ is made in the $x$ direction, resulting in:

$$
\delta \sigma \cdot \pi r^{2}+\tau \cdot 2 \pi r d x=0
$$

Then, the shear stresses are given by:

$$
\tau=-\frac{r}{2} \frac{d \sigma}{d x}
$$


By the differential of (18):

$$
\tau_{f}(x)=\frac{r \rho E_{f} \varepsilon}{2 \alpha L}
$$

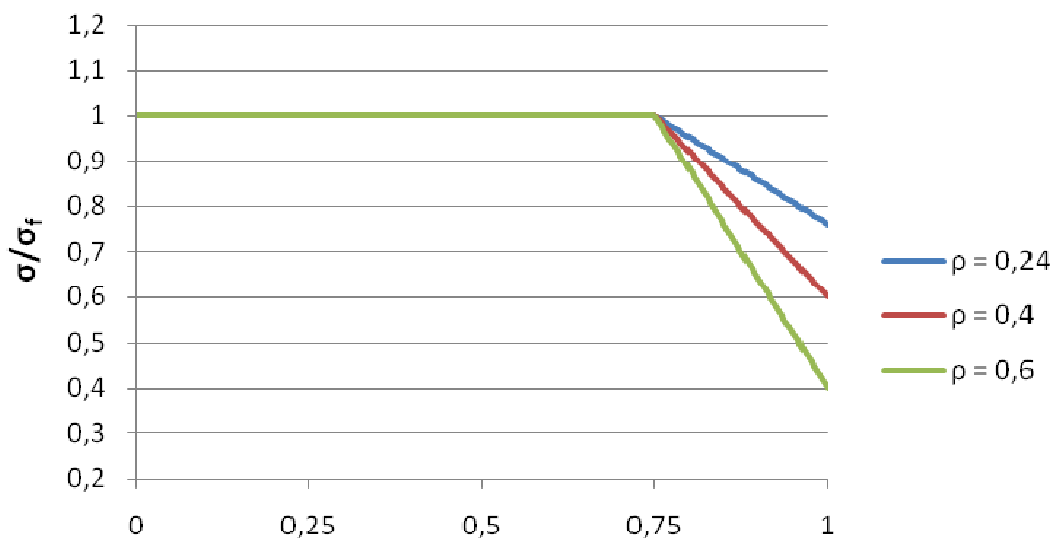

Distance from the fiber center (in.)

Figure 3: Stress distribution along the fibre for different matrix porosities. $\alpha=0.25$.

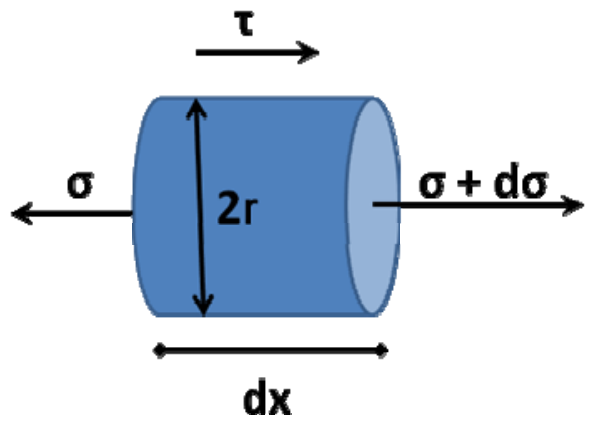

Figure 4: Force equilibrium in a fibre element whose length is $d x$.

With the stress distribution along the fibre, it is possible to calculate the average stress carried by the fibre in the composite, given by:

$$
\overline{\sigma_{f}}=\frac{1}{L} \int_{0}^{L} \sigma_{f}(x) d x
$$

For $\alpha \geq 1$, i.e. the fibre is shorter than the critical length:

$$
\overline{\sigma_{f}}=\frac{1}{L} \int_{0}^{L}-\frac{\rho E_{f} \varepsilon}{\alpha L} x+\left[1-\rho\left(1-\frac{1}{\alpha}\right)\right] E_{f} \varepsilon d x
$$




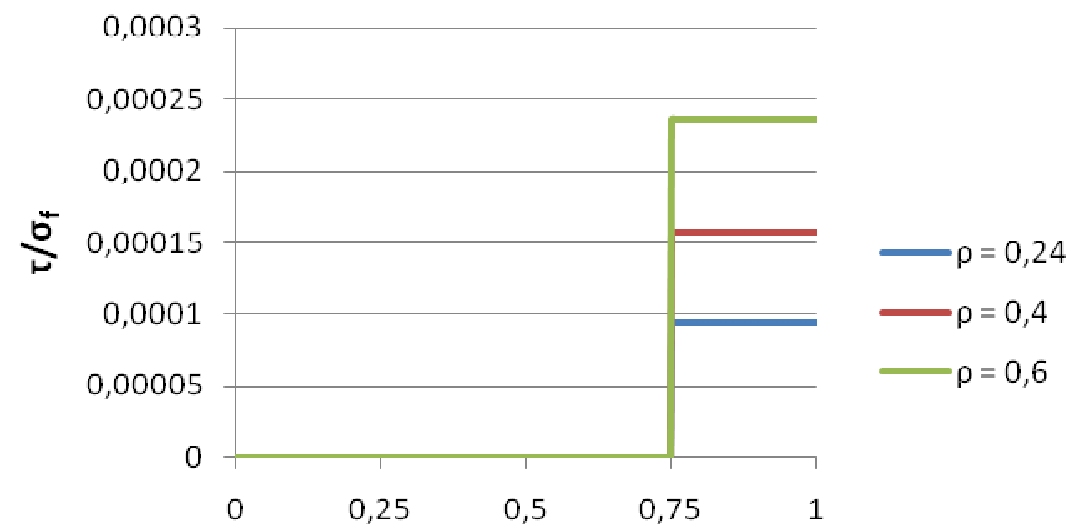

Distance from the fiber center (in.)

Figure 5: Shear stress distribution along the fibre for different matrix porosities. $\alpha=0.25$.

Then,

$$
\overline{\sigma_{f}}=\left[1-\rho-\frac{\rho}{2 \alpha}\right] E_{f} \varepsilon
$$

And for $0<\alpha<1$, i.e., the fibre is longer than the critical length:

$$
\overline{\sigma_{f}}=\frac{\int_{0}^{L-\alpha L} E_{f} \varepsilon d x+\int_{L-\alpha L}^{L} \sigma_{f}(x) d x}{L}
$$

Therefore:

$$
\overline{\sigma_{f}}=E_{f} \varepsilon\left(1-\frac{\rho \alpha}{2}\right)
$$

With the average stresses well defined, we can define the stresses in the ply longitudinal and transversal directions. When the matrix material is the same as the fibre, it is possible to write the elastic modulus of the matrix as a function of the fibre modulus:

$$
E_{m}=E_{f} e^{-b p}
$$

where $b$ is a shape factor that depends on the pore shape and distribution. 




Figure 6: Longitudinal resistance of the composite [6].

The stress on the transversal direction is proportional to the matrix maximum stress, given by:

$$
\sigma_{T}=\sigma_{m}=E_{f} \varepsilon e^{-b p}
$$

The stress on the longitudinal direction is given by the average value between matrix and fibre, based on the volumetric fractions of fibre and matrix:

$$
\sigma_{L}=\sigma_{m}\left(1-v_{f}\right)+\overline{\sigma_{f}} v_{f}=\left(1-v_{f}\right) E_{f} \varepsilon e^{-b p}+\overline{\sigma_{f}} v_{f}
$$

Therefore for $0<\alpha<1$ :

$$
\sigma_{L}=\left(1-v_{f}\right) E_{f} \varepsilon e^{-b p}+E_{f} \varepsilon\left(1-\frac{\rho \alpha}{2}\right) v_{f}
$$

And for $\alpha>1$ :

$$
\sigma_{L}=\left(1-v_{f}\right) E_{f} \varepsilon e^{-b p}+E_{f} \varepsilon\left[1-\rho-\frac{\rho}{2 \alpha}\right] v_{f}
$$




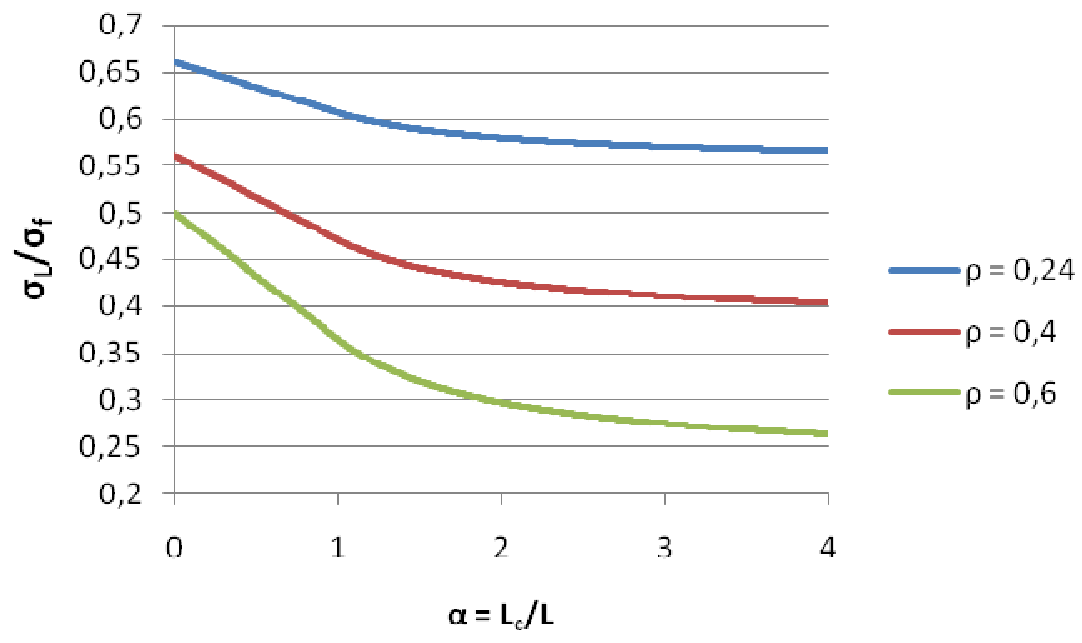

Figure 7: Longitudinal ply ultimate strength, for different $\alpha$.

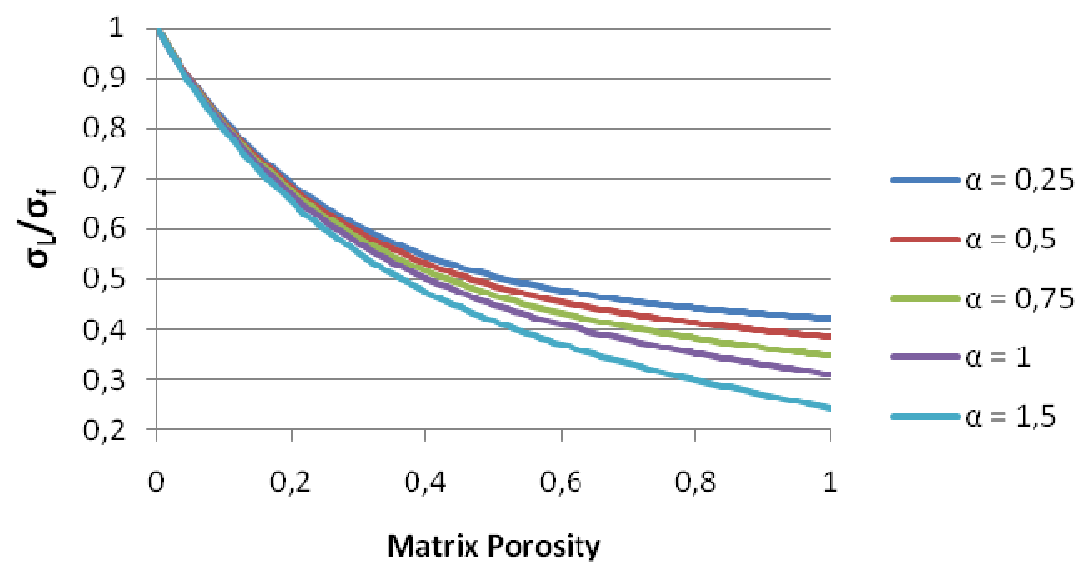

Figure 8: $\quad$ Longitudinal ply ultimate strength for different porosities.

\section{Conclusions}

A load transfer model was proposed to ceramic composites, which relates the matrix porosity and fibre length with the mechanical strength of such composites. It is hoped that the experimental validation of the model can be established or any changes aimed at increasing its accuracy can be made. 


\section{Acknowledgements}

The authors would like to thank the agencies CAPES (Coordenação de Aperfeiçoamento de Pessoal de Nível Superior), CNPq (Conselho Nacional de Desenvolvimento Científico e Tecnológico) and DFG (Deutsche Forschungsgemeinschaft), for funding this research under grant Bragecrim 015/09.

\section{References}

[1] Janssen, R., Scheppokat, S. \& Claussen, N., Tailor-made ceramic-based components - Advantages by reactive processing and advanced shaping techniques. Journal of the European Ceramic Society, 28, pp. 1369-1379, 2008.

[2] Janssen, R. \& Hotza, D. Low-cost and reliable production of oxide ceramic matrix composites, Bragecrim Project Proposal, December 2008.

[3] Wendorff, J., Janssen, R. \& Claussen, N., Saphirfaserverstärkung reaktionsgebundener oxider Keramiken. Verbundwerkstoffe und Werkstoffverbunde, ed. G. Ziegler, pp. 421-424, 1996.

[4] Lundberg, R. \& Eckerbom, L., Design and processing of Al-oxide composites. Ceramic Transactions, 58, pp. 95-104, 1995.

[5] Levi, C.G., Yang, J.Y., Dalgleish, B.J., Zok, F.W. \& Evans, A.G., Processing and performance of an all-oxide ceramic composite. Journal of the American Ceramic Society, 81, pp. 2077-2086, 1998.

[6] Casaril, A.; Gomes, E.R.; Soares, M.R.; Fredel, M.C. \& Al-Qureshi, H.A., Análise micromecânica dos compósitos com fibras curtas e partículas. Matéria, 12(2), pp. 408-419, 2007.

[7] Cox, H.L., The elasticity and strength of paper and other fibrous materials. British Journal of Applied Physics, 3, pp. 72-79, 1952 